\title{
In the time of corona - is it safe to delay treatment for prostate cancer?
}

\author{
Doao Paulo Pretti Fantin ${ }^{1}$ \\ (iD) Luis Cesar Fava Spessoto ${ }^{2}$ \\ (iD) Fernando Nestor Facio Junior ${ }^{2}$
}

1. Doutorando no Programa de Ciências da Saúde, Faculdade de Medicina (FAMERP), São José do Rio Preto, SP, Brasil 2. Professor de Urologia, Departamento de Urologia, Faculdade de Medicina (FAMERP/FUNFARME), São José do Rio Preto, SP, Brasil

Recently, the outbreak of a new disease denominated Coronavirus 2019 (COVID-19) occurred in Wuhan, China'; it rapidly spread to other regions of China and around the world ${ }^{2,3}$. This pandemic has implications on the continuity of treatment for patients, especially of cancer. There has been a shift in priorities. Human, medical, and paramedical resources are being directed at care for individuals infected by COVID-19. Moreover, measures to confine the population have led to the restriction of travel for appointments, exams, and clinical/surgical treatment.

Patients with prostate cancer, which is the most common form of cancer among men in Brasil, have a slow-moving condition ${ }^{4}$, and delaying treatment due to the pandemic is not likely to affect survival in the long term. In the current scenario, delaying surgical treatment for a localized, locally advanced disease is under discussion since it is an elective procedure and, therefore, among those that health authorities are advising to be delayed.

Most patients with prostate cancer are asymptomatic at the time of diagnosis and do not require urgent care. Morini et al. ${ }^{5}$ evaluated 908 patients submitted to radical prostatectomy and found no change in the oncological outcome when the procedure was delayed for six to 12 months after diagnosis in patients with low or intermediate risk. Likewise, O'Callaghan et at. ${ }^{6}$ found no worsening of overall survival or specific cancer in the Australian population with a delay of up to 8.8 months.

However, patients may attempt to persuade surgeons to opt for the surgical procedure even in this time of crisis. One should bear in mind that patients may be in the incubation period at the time of surgery. In a study involving 2,007 Chinese individuals hospitalized with COVID-19, Liang et al. ${ }^{7}$ found a significantly higher incidence of serious events such as death or admission to the ICU, requiring invasive mechanical ventilation, among individuals with a history of cancer than those without such a history (39\% vs. $8 \% ; \mathrm{P}=0.0003)$. In a study conducted by $\mathrm{Yu}$ et al. ${ }^{8}$, a total of 1,524 patients with cancer in Wuhan were analyzed and the authors estimated that those with cancer had a twofold higher risk of infection by COVID-19 in comparison to the general population. Therefore, cancer and hospitalization contribute to a greater risk of infection and worse outcomes.

In conclusion, even with the uncertainties regarding the period of isolation and the delaying of elective procedures, patients with localized prostate cancer of low risk have a low probability of progression of the neoplasm, even with the delay of surgical treatment. Therefore, such patients can wait up to six months.

\section{Conflicts of Interest}

The authors have no conflicts of interest to declare. 


\section{REFERENCES}

1. Zhu N, Zhang D, Wang W, et al. China Novel Coronavirus Investigating and Research Team. A Novel Coronavirus from Patients with Pneumonia in China, 2019. N Engl J Med. 2020;382(8):727-733.

2. Holshue ML, DeBolt C, Lindquist S, et al. Washington State 2019-nCoV Case Investigation Team. First Case of 2019 Novel Coronavirus in the United States. N Engl J Med. 2020;382(10):929-936.

3. Silverstein WK, Stroud L, Cleghorn GE, et al. First imported case of 2019 novel coronavirus in Canada, presenting as mild pneumonia. Lancet. 2020;395(10225):734.

4. Johansson JE, Andrén O, Andersson SO, et al. Natural history of early, localized prostate cancer. JAMA. 2004;291(22):2713-9.
5. Morini MA, Muller RL, de Castro Junior PCB, et al. Time between diagnosis and surgical treatment on pathological and clinical outcomes in prostate cancer: does it matter? World J Urol. 2018;36(8):1225-1231.

6. O'Callaghan ME, Shi Z, Kopsaftis T, et al. Prostate cancer outcomes and delays in care. Int Urol Nephrol. 2017;49(3):449-455.

7. Liang W, Guan W, Chen R, et al. Cancer patients in SARS-CoV-2 infection: a nationwide analysis in China. Lancet Oncol. 2020;21(3):335-337.

8. Yu J, Ouyang W, Chua MLK, et al. SARS-CoV-2 Transmission in Patients With Cancer at a Tertiary Care Hospital in Wuhan, China. JAMA Oncol. 2020 Mar 25. doi: 10.1001/jamaoncol.2020.0980. [Epub ahead of print]. 